\title{
High-Frequency EEG Variations in Children with Autism Spectrum Disorder during Human Faces Visualization
}

\author{
Celina A. Reis Paula, ${ }^{1,2}$ Camille Reategui, ${ }^{1}$ Bruna Karen de Sousa Costa, ${ }^{3}$ \\ Caio Queiroz da Fonseca, ${ }^{3}$ Luana da Silva, ${ }^{4}$ Edgard Morya, ${ }^{1}$ and Fabricio Lima Brasil ${ }^{1}$ \\ ${ }^{1}$ Edmond and Lily Safra International Institute of Neuroscience, Santos Dumont Institute, \\ Rod. RN 160, Km 03, No. 3003, 59280-000 Macaiba, RN, Brazil \\ ${ }^{2}$ Anita Garibaldi Center for Education and Research in Health, Santos Dumont Institute, \\ Rod. RN 160, Km 02, No. 2010, 59280-970 Macaiba, RN, Brazil \\ ${ }^{3}$ Electrical Engineering Department, Federal University of Campina Grande (UFCG), 882 Aprígio Veloso St, \\ 58429-900 Campina Grande, PB, Brazil \\ ${ }^{4}$ Electrical Engineering Department, Federal University of Santa Maria (UFSM), 1000 Roraima Av., 97105-900 Santa Maria, RS, Brazil
}

Correspondence should be addressed to Celina A. Reis Paula; larissaangelia2010@gmail.com

and Fabricio Lima Brasil; brasil@isd.org.br

Received 1 May 2017; Revised 27 June 2017; Accepted 27 July 2017; Published 6 September 2017

Academic Editor: Victor H. C. de Albuquerque

Copyright (C) 2017 Celina A. Reis Paula et al. This is an open access article distributed under the Creative Commons Attribution License, which permits unrestricted use, distribution, and reproduction in any medium, provided the original work is properly cited.

\begin{abstract}
Autism spectrum disorder (ASD) is a neuropsychiatric disorder characterized by the impairment in the social reciprocity, interaction/language, and behavior, with stereotypes and signs of sensory function deficits. Electroencephalography (EEG) is a well-established and noninvasive tool for neurophysiological characterization and monitoring of the brain electrical activity, able to identify abnormalities related to frequency range, connectivity, and lateralization of brain functions. This research aims to evidence quantitative differences in the frequency spectrum pattern between EEG signals of children with and without ASD during visualization of human faces in three different expressions: neutral, happy, and angry. Quantitative clinical evaluations, neuropsychological evaluation, and EEG of children with and without ASD were analyzed paired by age and gender. The results showed stronger activation in higher frequencies (above $30 \mathrm{~Hz}$ ) in frontal, central, parietal, and occipital regions in the ASD group. This pattern of activation may correlate with developmental characteristics in the children with ASD.
\end{abstract}

\section{Introduction}

Autism spectrum disorder is a neurodevelopmental disorder with well-defined diagnostic criteria such as communications/social interaction and behavior deficits, with restricted and repetitive interests and activities $[1,2]$. These social communication disturbances present a complex and heterogeneous pattern and for that reason fit in a spectrum and may present various patterns of severity in symptoms or evolution profile. The initial publications about the autism spectrum disorder were only in the form of case reports [3, 4]. In 1966, the first epidemiological study suggested the occurrence of
4.5 children with ASD per 10,000 inhabitants in the age range of 8 to 10 [5]. However, further studies realized in Europe, United States, Canada, and Japan suggest an increase in this incidence, with values of 10,30 , or even 60 cases per 10,000 inhabitants [6]. Nowadays, the ASD seems to affect approximately 1 in 68 children, most common among boys [7]. There is a suggestion of increased incidence and prevalence of ASD worldwide [8]. This increase is probably due to the changes in the diagnostic criteria [9], emergence and development of diagnostics, early intervention services, and more direct approaches related to the diagnosis of the disorder [10]. 
TABle 1: Participants demographics.

\begin{tabular}{lcccccccc}
\hline & \multicolumn{3}{c}{ Experimental group (ASD) } & & & \multicolumn{3}{c}{ Control group } \\
& Age(yrs) & Gender & Language structure & Age at diagnosis (yrs) & & Age & Gender & Language structure \\
\hline Subject 1 & 10 & Male & Sentences & 5 & Subject 9 & 10 & Male & Verbal fluency \\
Subject 2 & 12 & Female & Phrases & 5 & Subject 10 & 12 & Female & Verbal fluency \\
Subject 3 & 6 & Male & Word & 2 & Subject 11 & 6 & Male & Verbal fluency \\
Subject 4 & 7 & Male & Sentences & 3 & Subject 12 & 7 & Male & Verbal fluency \\
Subject 5 & 8 & Male & Sentences & 4 & Subject 13 & 8 & Male & Verbal fluency \\
Subject 6 & 9 & Male & Word & 4 & Subject 14 & 9 & Male & Verbal fluency \\
Subject 7 & 5 & Male & Sentences & 3 & Subject 15 & 5 & Male & Verbal fluency \\
Subject 8 & 11 & Male & Sentences & 4 & Subject 16 & 11 & Male & Verbal fluency \\
\hline
\end{tabular}

The ASD diagnostics are realized according to the observation of initial symptoms in early childhood with impairments in their daily functionality [2], neurodevelopment, children's behavioral characteristics, and objective clinical analysis. The clinical signs should fulfill the diagnostic criteria described in the Diagnostic and Statistical Manual for Autism (DSM-V). Questionnaires, checklists, and diagnostic scales support the evaluation and confirm the diagnosis [11]. Children with ASD can present a heterogeneous clinical picture, in which the behavioral symptoms prevail. Such facts boost a search for biological markers of the disorder using different tools, for example, the electroencephalogram (EEG), the EyeTracker, the Functional and Structural Magnetic Resonance Imaging (fMRI or MRI), Positron Emission Tomography (PET), and Computerized Tomography (CT) based on emission of single photon (SPECT) [12]. These tools have been increasingly exploited in scientific research.

Abnormal EEG activity of the epileptic type occurs in $30 \%$ of the cases of ASD, even without epileptic seizures [13]. Paroxysmal discharges and slow focal activity were registered in the temporal region in EEG of patients with the disorder, especially those with developmental regression [14]. Although EEG can provide important information about brain function during resting and stimulation, the qualitative visual signal analysis of time domain seems to be insufficient to consider a pathognomonic pattern for the ASD [15]. Techniques for quantitative analysis, such as the Fourier Transform, favor a more detailed frequency analysis by bandwidth and its characteristics. EEG analysis of children with ASD shows differences in brain electrical signals compared to children without ASD $[5,16]$. A reduction in the power spectrum in the alpha bandwidth $(4-8 \mathrm{~Hz})$ was previously observed in EEGs of children with ASD during rest [14]. Also, hemispheric asymmetry of activity has been shown, such as greater activity in the left frontal lobe when observing happy facial expressions with smiles [17] and decreased activity in the same region when observing facial expressions of fear [18]. It was also observed that a theta power spectrum in the frontal midline (Fm) is related to emotional states. Sammler and colleagues proposed that pleasant emotions (opposed to unpleasant) are related to the increase in the theta power density in the Fm [19]. Thus, in light of previous studies and considering the behavioral component in the diagnosis of ASD, it is expected that the use of the EEG to access neural activities elicited by social stimuli has the potential of providing a quantitative analysis of impairment in social interaction activities of this group [20].

Neuropsychological patterns verified in ASD might suggest the involvement of other brain regions. The difficulty in maintaining attentional focus or the behavior of paying attention to a face or object details instead of the whole picture might be related to difficulty in shared attention and in the executive function, capabilities that involve the frontal region.

The current study aims to show quantitative differences in the frequency spectrum pattern between the EEG of children with and without ASD before and during the observation of human faces. We believe that finding these differences can lead to a better understanding of how these children could potentially be better stimulated and taught using their respective preference of human faces or figure faces. We hypothesize that social interaction impairment present in children with ASD, when compared with children without ASD, can occur due to a deficit in the visual processing of human faces. In addition, we also hypothesize that the brain's electrical activity presents a different quantitative pattern in the power spectrum in the bandwidths during the observation of stimulus such as human faces with different emotion expressions.

\section{Materials and Methods}

2.1. Participants. This research was authorized by the Ethics Committee of the Federal University of Rio Grande do Norte (CAAE 46207015.0.0000.5537). The consent form was read and explained to the parents, and after their agreement, they signed the consent form. EEG data were recorded at the Anita Garibaldi Center for Education and Research in Health (CEPS). Sixteen children participated in the study: eight with ASD and 8 without ASD. All of them were from the same metropolitan region and were paired by age (Table 1). The age of the participants varied from 5 to 12 years 


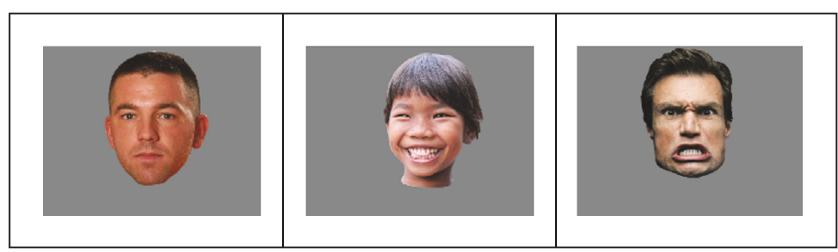

FIGURE 1: Images showing human faces with neutral, happy, and angry expressions.

$(M=8.44, \mathrm{SD}=2.24)$, and only two (one of each group) were female. The inclusion criteria for the group with ASD were the presence of the diagnostics realized by a neuropediatrician after neuropsychological evaluation. For the children of the control group, it was necessary to test for intellectual disability with the ASD diagnostics, also by the same team. Children with epileptic seizures in the last 3 years did not participate in the research. Subjects who did not cooperate to perform the exam also were excluded and respective data were not included in the analysis.

The ASD diagnostics were realized by a neuropediatric physician, considering the fulfillment of the diagnostics criteria of the DSM-V, the neuropsychological evaluation with the childhood autism rating scale (CARS), an IQ test, and language evaluation.

\subsection{Experimental Setup}

2.2.1. EEG. EEG-1200 (Neurofax, Nihon Kohden, Tokyo, Japan) was used for noninvasive brain electrophysiological signal acquisition (sampling rate $1.000 \mathrm{~Hz}$ ) with 22 electrodes (Ag/AgCl disk electrode, 10/20 distribution with ear lobes ground) positioned with previous scalp preparation (cleaned with neutral soap, dry and no hair creams or hair products, and impedance lower than $5 \mathrm{k} \Omega$ ). EEG data were recorded and synchronized (StimTracker ST-100, Cedrus, USA) with visual stimulation and eye-tracking.

2.2.2. Visual Stimuli. Visual stimuli with 30 human faces were presented on a grey background (Figure 1). The faces were paired by expression and classified into three groups: 10 neutral, 10 happy, and 10 angry.

All stimuli were size dimensioned and standardized to keep equivalent distances between the eyes, mouth, and nose in a central square area of the $17^{\prime \prime}$ LCD screen $(100 \mathrm{~Hz}$, Samsung) $60 \mathrm{~cm}$ from the participant's eyes. E-Prime ${ }^{\circledR} 2.0$ software (Psychology Software Tools, Inc., USA) presented in a sequence (happy-neutral-angry faces), interspersed by a fixation point in the center of the screen. Each face was presented for 3 seconds with 0.5 to $1.0 \mathrm{~s}$ of interval controlled by Mangold Vision 3.9 (Mangold International GmbH, Germany) software in programmed sequence and time.

2.2.3. Eye-Tracking. An Eye-Tracker (Eye-Tech TM3 $60 \mathrm{~Hz}$, Mesa, USA) was positioned under the screen to ensure the participants were looking at fixation point and visual stimuli during the task.

2.2.4. Task. Participants sat comfortably in a quiet dimmed room $60 \mathrm{~cm}$ from the LCD screen with EEG electrodes. They were instructed to keep the eyes at the fixation point and look at the image during the presentation. After eye-tracking calibration, EEG recording started two minutes before the visual stimulation.

2.3. Data Preprocessing. EEG data underwent preprocessing with a custom MATLAB (Mathworks, USA) script, EDF Browser (C Copyright 2017 Teunis van Beelen), and Python (Python Software Foundation).

2.3.1. EDF Browser. EDF Browser converted raw EEG data to ASCII format compatible with Python and MATLAB. The epochs to be analyzed were correctly separated according to the stimuli marker registered during the experiment in order to organize files. Thus, for each subject, three files were generated containing, respectively, all epochs of happy, neutral, and angry faces, for all EEG channels. These files were then processed by Python Programming Language.

2.3.2. Python Programming Language. Python libraries for data manipulation and analysis were used to organize sets of trials with all subjects of each group and separate channels. This resulted in.csv files with raw EEG data of subjects for each type of stimuli and for each channel. Thereafter, these files were processed by MATLAB.

2.3.3. MATLAB. A MATLAB code designed a 6th-order bandpass Butterworth filter with a lower cutoff frequency of $1 \mathrm{~Hz}$ and a higher cutoff frequency of $100 \mathrm{~Hz}$. A Notch filter removed the frequency component of the electrical grid $(58-62 \mathrm{~Hz})$. Moreover, a technique for detecting spectral perturbation related to the event (ERSP) was implemented. The ERSP consists of a tool to observe the variations on local field potentials related to the event by calculating the mean and the standard deviation of the EEG signal in order to normalize the signal prior to the event. Each epoch has $-500 \mathrm{~ms}$ before stimulus presentation and $+3.500 \mathrm{~ms}$. Spectral features related to the event were calculated for this time window. After that, the same procedure was done in the event epochs, where they were analyzed with the ERSP tool 

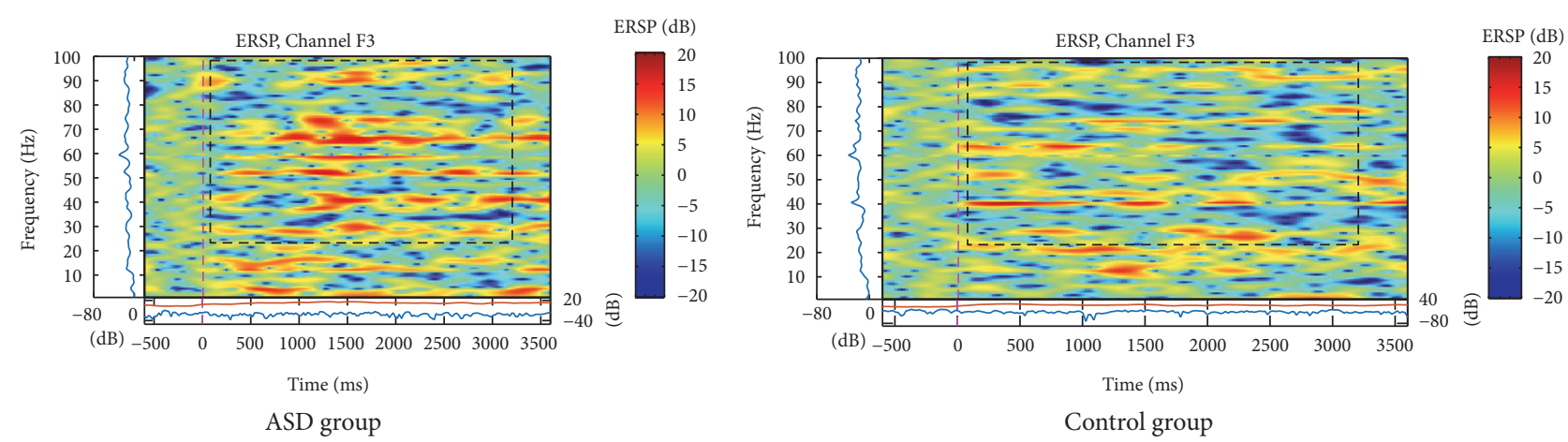

(a)
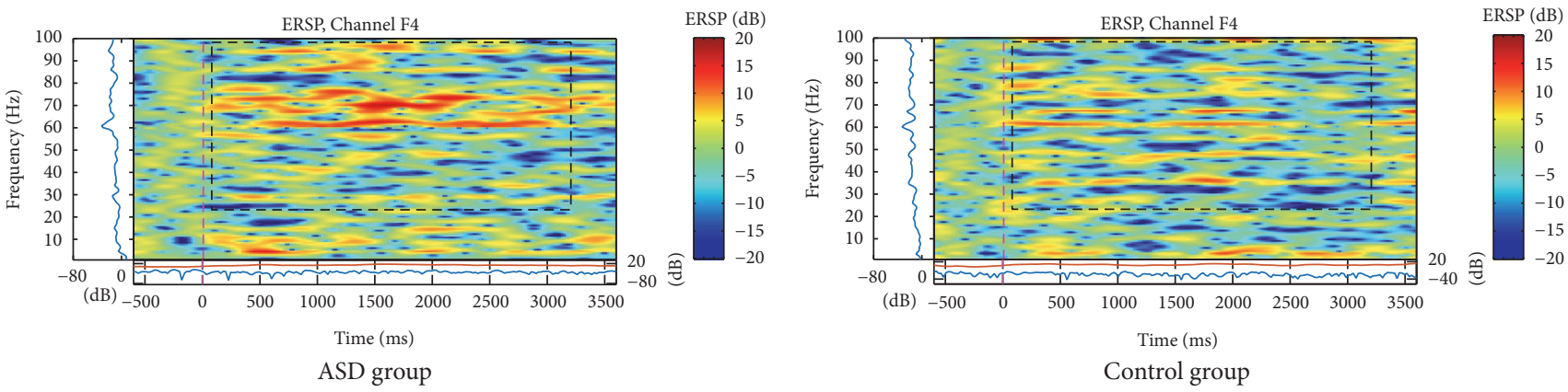

(b)

FIGURE 2: ERSP of (a) F3 and (b) F4 electrodes for the total mean of samples for angry expression signal in an interval of 3 seconds (plus a basal activity of $500 \mathrm{~ms}$ before and after an event) for ASD and control groups that showed significant variations or spectral perturbations. The ERSP image in the upper panel presents the ERSP (Event-Related Spectral Perturbation) data in dB, with mean baseline spectral power subtracted at each time in the epoch. The upper left marginal panel presents mean spectral power during the baseline period (blue). The marginal panel under the ERSP image shows the maximum (red) and minimum (blue) ERSP values relative to baseline power for each frequency.

in order to calculate the variation or spectral perturbation related to the event occurrence.

\section{Data Analysis and Results}

3.1. Clinical Results. Group ASD presented verbal patterns, with $37.5 \%$ able to speak few words and sentences, but not enough to maintain a dialogue. Control group also presented verbal patterns, with $100 \%$ able to speak few words, sentences, and adequate conversation. All children were attending school, $50 \%$ of group ASD were literate, and $50 \%$ were in the presyllabic stage. In the control group, only one subject (4 years old) was in the presyllabic stage and the others (87\%) were literate.

The ASD's diagnostic age was 4.77 years $(S D=2.30)$. The ASD parents' age was $40.37 \pm 4.59$ years and the control group parent's age was $34.62 \pm 8.17$ years. The predominant parent's education level was college $(43.8 \%)$ for the ASD group and high-school (56.3\%) for the control group. The average income for both groups was between 1 and 5 minimum salaries (75\%).

3.2. EEG Power Spectral Density. EEG Power Density Spectrograms were generated using MATLAB for the mean of
ASD and control group. Differences between ASD and control groups were observed in power spectrum parameters, with stronger activation for Gamma band (above $30 \mathrm{~Hz}$ ), and along frontal, central, parietal, and occipital electrodes.

For the ASD group, the major activation was verified in the Fp and F electrodes for frequencies above $20 \mathrm{~Hz}$, in the parietal and central electrodes for frequencies between 40 and $50 \mathrm{~Hz}$, and in the occipital electrodes for frequencies above $40 \mathrm{~Hz}$. In a lower incidence, there was also an increase of slow activity (below $8 \mathrm{~Hz}$ ) in the frontal, parietal, and occipital regions. In general, the major activation occurred in the left brain hemisphere for the ASD group.

Graphics were generated with the mean of each group according to the type of stimulus (neutral, happy, and angry expressions) and only graphics that showed differences between the ASD and control groups were presented. In this case, the distinctness of the evaluation responses was confirmed in the $\mathrm{F} 3 / \mathrm{F} 4, \mathrm{C} 3 / \mathrm{C} 4, \mathrm{P} 3 / \mathrm{P} 4$, and $\mathrm{O} 1 / \mathrm{O} 2$ electrodes. In frontal electrodes, the activation was bigger in the ASD group and mainly in higher frequencies (above $30 \mathrm{~Hz}$ ), but it was also increased in the theta and delta bands for angry faces, as shown in Figures 2(a) and 2(b).

In parietal electrodes, the differences between the groups were also verified. ASD group presented more activation in 

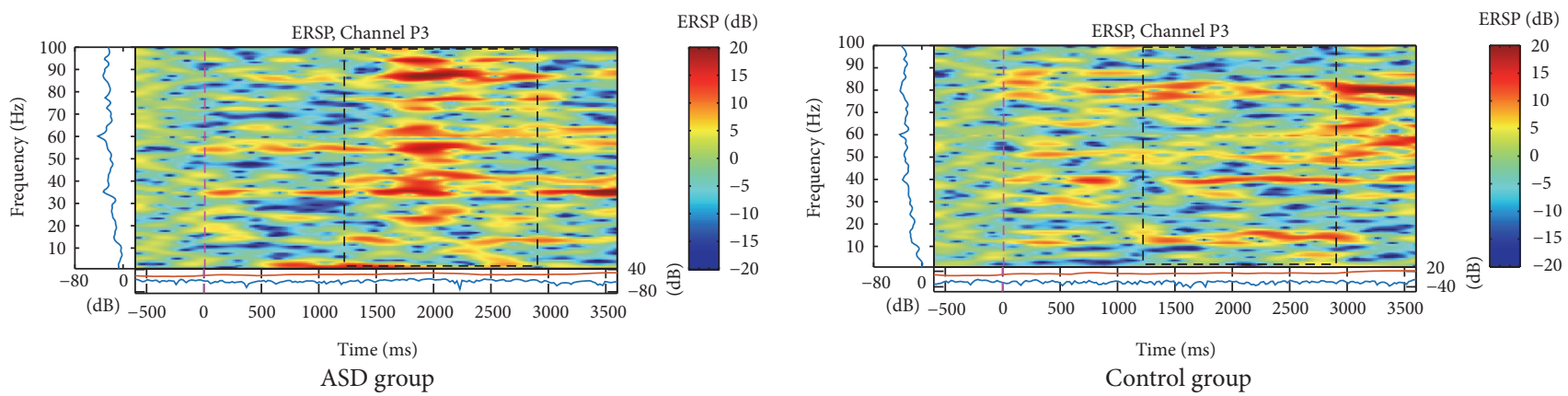

(a)
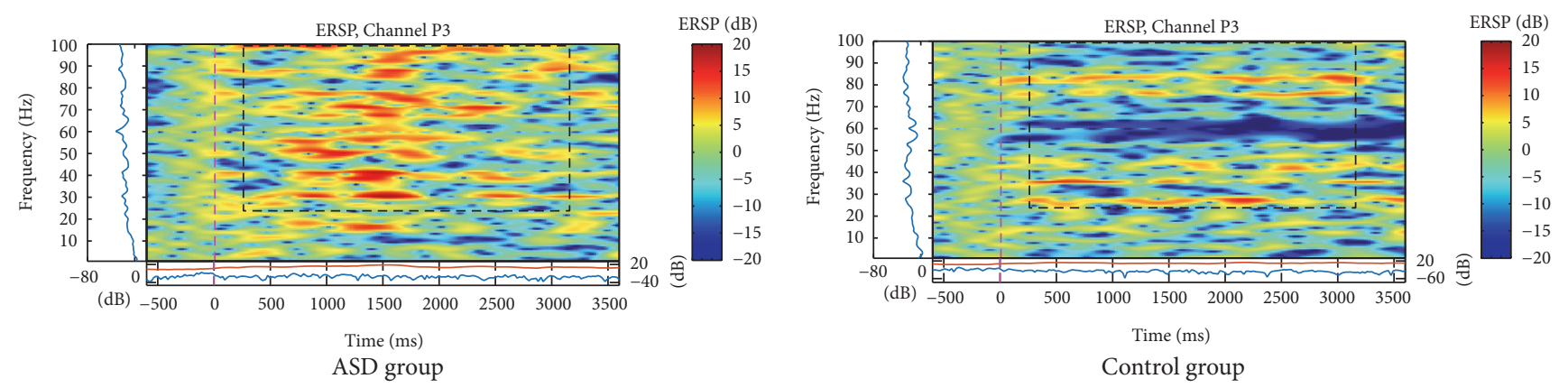

(b)
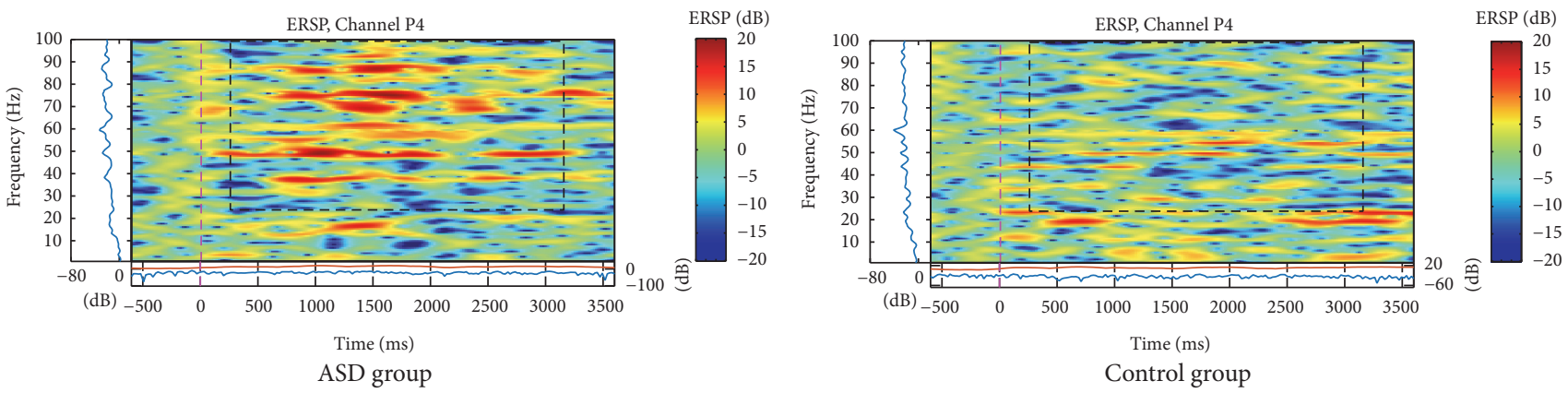

(c)

Figure 3: ERSP of P3 (a, b) and P4 (c) electrodes for total mean of samples of the neutral (a), and angry (b), and (c) faces signals in interval of 3 seconds (plus a baseline activity of $500 \mathrm{~ms}$ before the event) for ASD and control groups that showed significant spectral perturbations. In ERSP image, the upper panel presents the ERSP (Event-Related Spectral Perturbation) data in dB, with mean baseline spectral power (in dB) subtracted at each time in the epoch. Upper left marginal panel presents the mean spectral power during the baseline period (blue). Marginal panel under the ERSP image shows the maximum (red) and minimum (blue) ERSP values relative to baseline power at each frequency.

slow frequencies (below $5 \mathrm{~Hz}$ ) for neutral faces on electrode P3 and higher frequencies (above $30 \mathrm{~Hz}$ ) for neutral faces as well as for angry faces, having a symmetric pattern only for the latter (Figure 3). Analysis of variance showed a main effect for Gamma band on electrodes P3, $F(1,1118)=9.55$, $p<.000$, and $\mathrm{P} 4, F(1,1118)=6.20, p<.000$. Post hoc independent-samples $t$-test indicated that scores for electrode $\mathrm{P} 3$ were significantly higher for the ASD group $(M=$ $.255, \mathrm{SD}=.341)$ than for the control group $(M=.071, \mathrm{SD}$ $.200), t(1118)=11.1, p<.001$.

The C3 and C4 electrodes showed higher bilateral activation in all frequency bands in the ASD group to the stimulus of neutral (Figure 4) and angry (Figure 5) faces. Analysis of variance showed a main effect for Gamma band on electrodes C3, $F(1,1118)=8.36, p<.000$, and C4, $F(1,1118)=75.1$, $p<.000$. Post hoc independent-samples $t$-test indicated that scores for electrode C3 were significantly higher for the ASD group $(M=.287, \mathrm{SD}=.340)$ than for the control group $(M=.114, \mathrm{SD} .266), t(1118)=9.47, p<.001$, and the same pattern was found for electrode C4, where the ASD group were higher $(M=.255, \mathrm{SD} .327)$ than for the control group $(M=.103, \mathrm{SD}=.253), t(1118)=8.67, p<.001$.

The activation was similar for parietal and central regions on neutral faces (similarity just on the left side) and mainly 

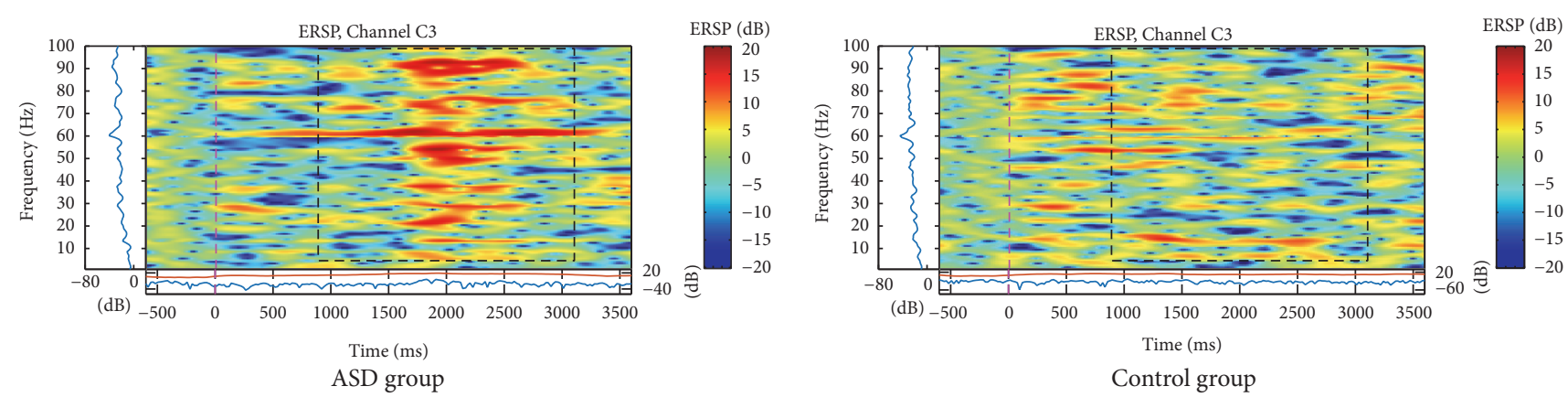

(a)
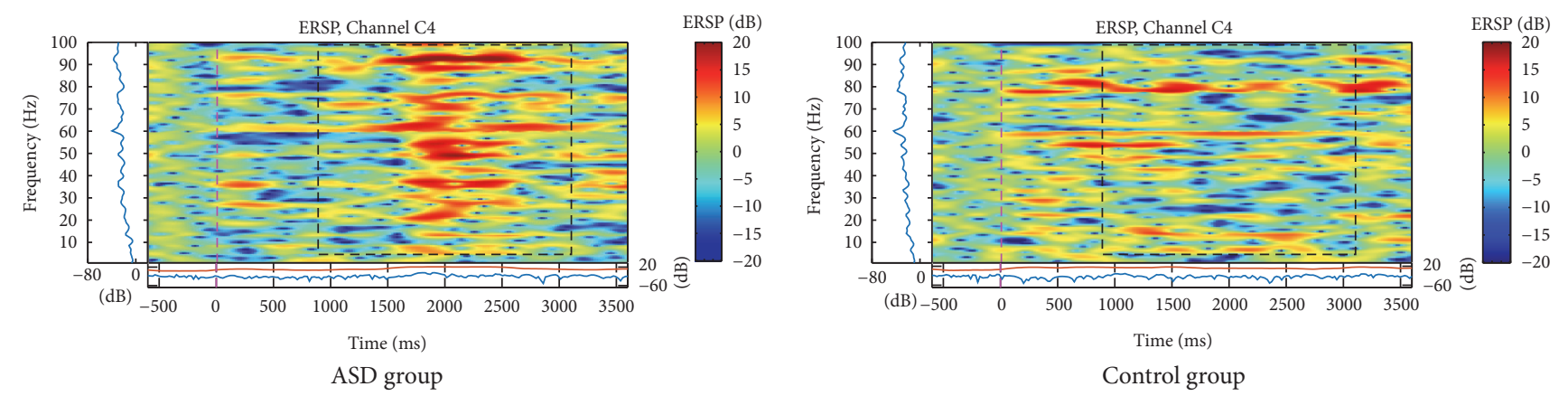

(b)

FIGURE 4: ERSP of (a) C3 and (b) C4 electrodes for the total mean of samples of the neutral faces signals in the interval of 3 seconds (plus a basal activity of $500 \mathrm{~ms}$ before the event) for the ASD and control groups that showed significant spectral perturbations. In the ERSP image, the upper panel presents the ERSP (Event-Related Spectral Perturbation) data in $\mathrm{dB}$, with mean baseline spectral power (in dB) subtracted at each time in the epoch. The upper left marginal panel presents the mean spectral power during the baseline period (blue). The marginal panel under the ERSP image shows the maximum (red) and minimum (blue) ERSP values relative to baseline power at each frequency.

for angry faces (bilateral similarity). Neutral faces showed an increase of spectral power between 10 and $100 \mathrm{~Hz}$, mostly at 2 seconds, in C3, C4 (Figure 4), and P3 channels (Figure 3(a)), but only for ASD group.

For angry face stimulus, spectral power was higher in higher frequencies (between 20 and $50 \mathrm{~Hz}$ ), on C3 and C4 channels, at 1.5 seconds (Figure 5). The P3 (Figure 3(b)) and P4 (Figure 3(c)) channels similarly showed higher power in higher bands (above $60 \mathrm{~Hz}$ ).

For the happy face stimulus, the C3 and C4 channels of the control group presented activation in frequencies under $8 \mathrm{~Hz}$ from the beginning of the stimuli till 2 seconds later (Figure 6). Simultaneously, there was an activation in higher frequencies (above $30 \mathrm{~Hz}$ ). However, in ASD group, there was desynchronization mainly in higher frequencies (above $30 \mathrm{~Hz}$ ) until the end of the stimulus.

Differences occurred in occipital electrodes in the ASD group for the three types of faces, and there was a bilateral desynchronization, but mainly in the left hemisphere for neutral faces (Figure 7(c)). Also, on the left hemisphere, there were differences between happy and angry faces due to a significant increase in spectral power in all frequency bands, mainly the higher bands right after the happy pattern stimulus (Figures 7(a) and 7(b)) and during the angry pattern stimulus (Figure 8). In this case, significant activation occurred to the right hemisphere also in the ASD group before happy faces and there was no asymmetry in the control group (Figures 7 and 8).

\section{Discussion}

In this study, EEG analyses in children with ASD diagnostics paired with children without ASD were compared during the observation of faces with neutral, happy, and angry expression. Children with ASD presented stronger power spectrum in higher frequencies than the control group for some brain areas. Differences were more evident in occipital and centerparietal regions. Central regions showed a similar pattern to parietal, with same power activation in the same time that the stimulus was presented. Given the clinical evidence of an emotional, cognitive, and behavioral deregulation [21], one of the possible explanations is a perturbation in brain function with stronger or weaker connectivity between areas like the amygdala and prefrontal ventrolateral cortex and orbitofrontal cortex [21].

Developmental psychology suggests that children imitate facial gestures from an early age. This premature imitation might be related to a direct connection from a visual input 

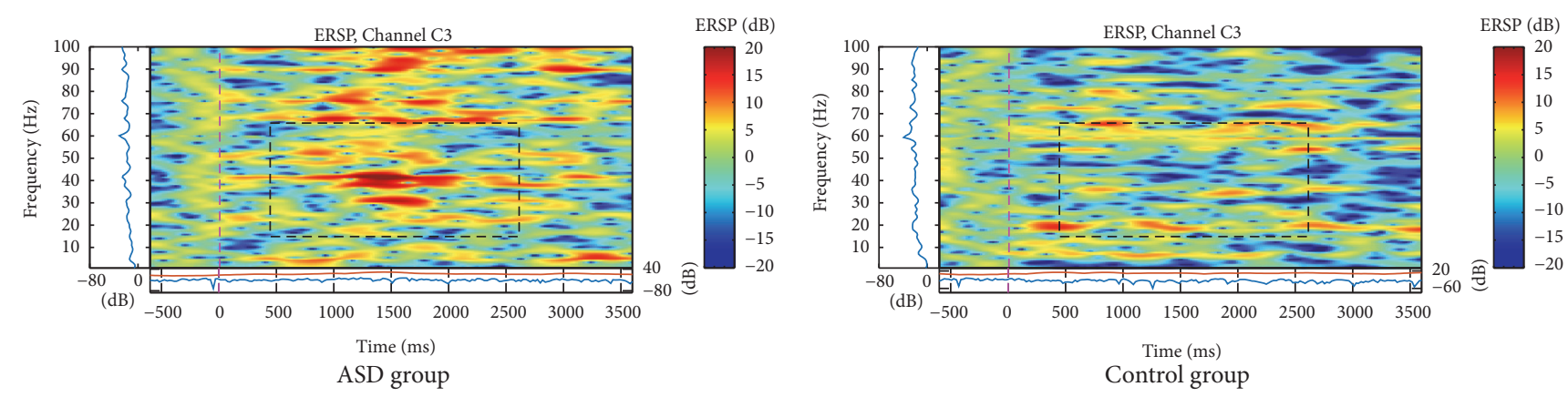

(a)
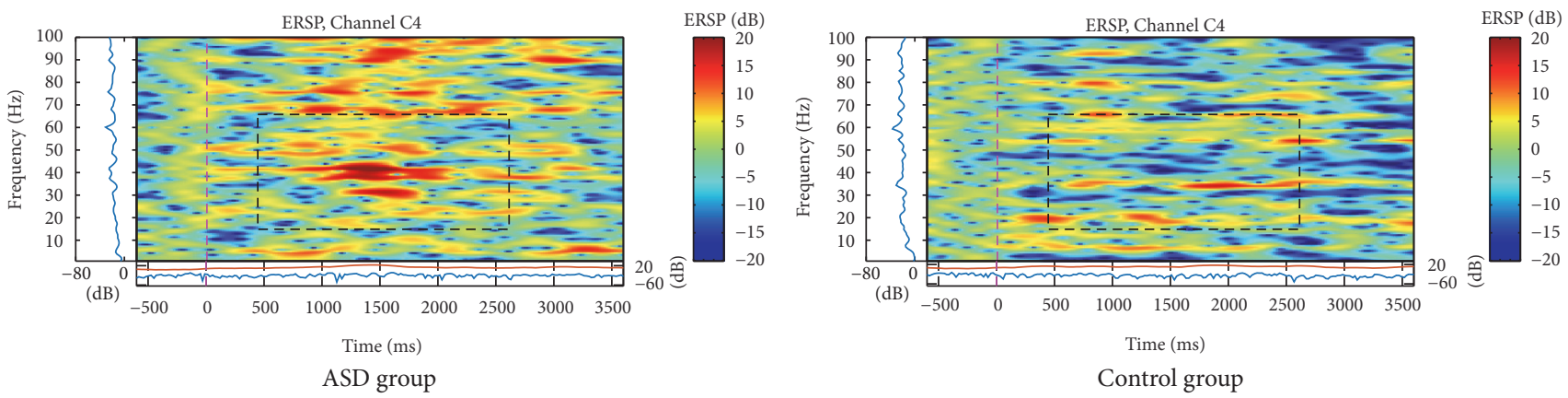

(b)

FIgURE 5: ERSP of (a) C3 and (b) C4 electrodes for the total mean of samples of the angry faces signals in the interval of 3 seconds (plus a basal activity of $500 \mathrm{~ms}$ before the event) for the ASD and control groups that showed significant variations or spectral perturbations. In the ERSP image, the upper panel presents the ERSP (Event-Related Spectral Perturbation) data in $\mathrm{dB}$, with mean baseline spectral power (in $\mathrm{dB}$ ) subtracted at each time in the epoch. The upper left marginal panel presents the mean spectral power during the baseline period (blue). The marginal panel under the ERSP image shows the maximum (red) and minimum (blue) ERSP values relative to baseline power at each frequency.

to a specific motor output [22]. This function is related to the systems of mirror neurons [23]. Failures in this system may be related to the social cognition deficits of ASD. This system suggests a strong relationship between action and intention recognition with social cognition, since it seems to regulate premotor cortex during observation action [24].

It is believed that mirror neurons form a system localized in the inferior parietal lobe, inferior frontal gyrus, superior temporal sulcus, and parietal-frontal lobe. Mirror neurons can be activated by visual stimulus. It was observed that, for visual stimuli indicating action, children with ASD present stronger activation of primary motor areas when compared to activation in the supplementary motor area [25].

Formation of the local network is needed for typical development in childhood and, after that, distribution of neural network in the teenage years and adult phase [26]. ASD children seem to have an atypical organization of the primary motor cortex, resulting in a subconnectivity with weak and short functional reach [26]. These subnetworks might generate execution loss of gestures linked to communication with consequent influence on social behavior [26].

Children with ASD can present differences in brain activities in visual-spatial processing related to object recognition (occipital, temporal, and ventral) and localization of objects in space (parietal, temporal, and dorsal). Communication failures between the dorsal and ventral pathways can harm the visual processing [27] and a lesion in these areas can lead to visual negligence and spatial distortions of body movements [27]. Various neurophysiological studies [7, 28-30] have tried to correlate these clinical symptoms through the demonstration of deficiencies or functional abnormalities of neural networks.

The findings of this study can be correlated to the clinical signs of children with ASD. The hypothesis is that, in ASD children, a deficit in facial expression processing will occur, with consequent failure in the storage for posterior access. The primary visual and primary motor areas are well activated, with some influence of decoding of the parietal lobe. Because of that, a bigger power spectrum might have occurred practically of the same pattern of central and parietal regions. It is believed that, in $\mathrm{C} 3$ and $\mathrm{C} 4$ electrodes during the stimuli of neutral and angry face, the presence of excessive fast rhythm in the ASD group when compared to the control group is probably due to the bottom-up activation. These failures of connectivity promote a mirror neurons system behavior similarly to the imitation of immature children that presents the direct conversion of the stimulus input (primary visual cortex) and motor output (motor cortex). In contrast, 

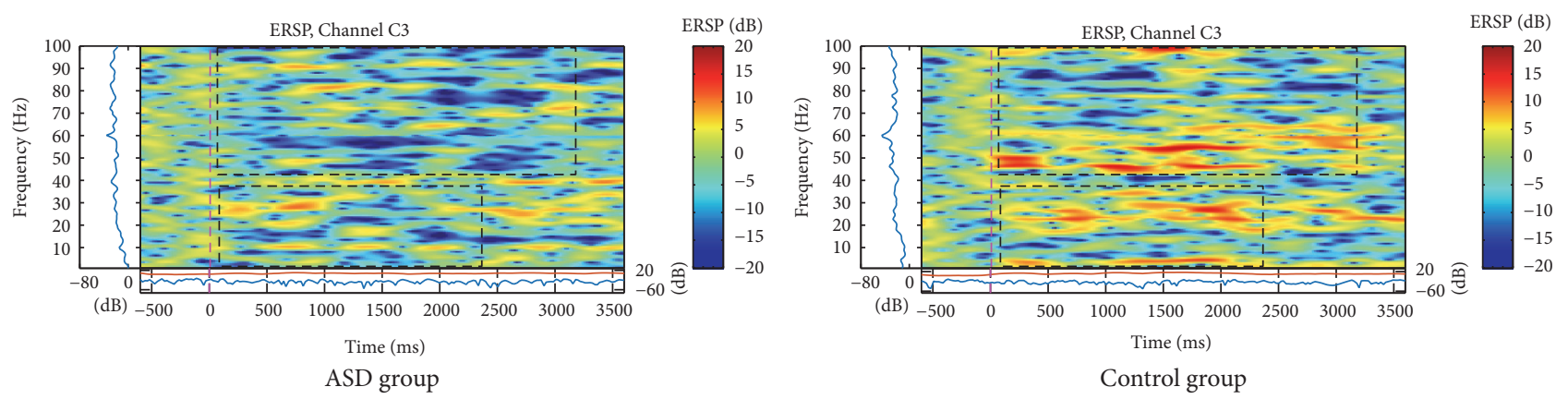

(a)
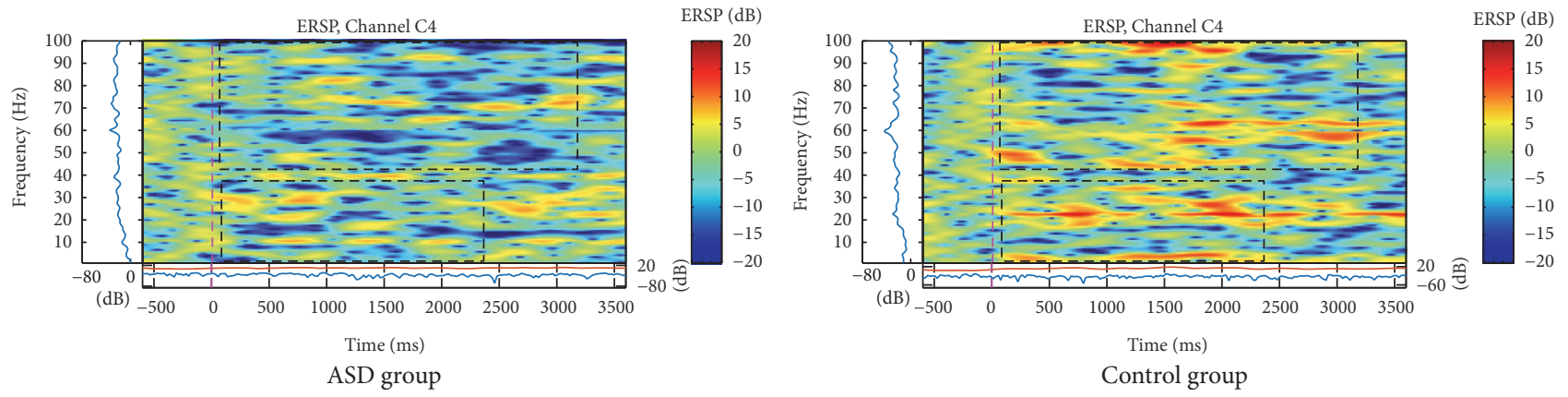

(b)

FiguRE 6: ERSP of (a) C3 and (b) C4 electrodes for the total mean of samples of the happy faces signals in the interval of 3 seconds (plus a basal activity of $500 \mathrm{~ms}$ before the event) for the ASD and control groups that showed significant variations or spectral perturbations. In the ERSP image the upper panel presents the ERSP (Event-Related Spectral Perturbation) data in dB, with mean baseline spectral power (in $\mathrm{dB}$ ) subtracted at each time in the epoch. The upper left marginal panel presents the mean spectral power during the baseline period (blue). The marginal panel under the ERSP image shows the maximum (red) and minimum (blue) ERSP values relative to baseline power at each frequency.

children with ASD have visual tracking patterns with greater fixation in regions important for emotional expressiveness (such as eyes and mouth) when exposed to happy face stimuli [28]. This behavior may help a more emotional maturity processing and therefore, a cerebral hypoactivation in $\mathrm{C} 3$ and $\mathrm{C} 4$ for fast frequency bands in this group in relation to the control was observed in this study. Similarly, there is a flaw in the rendering of faces with emotion mainly for neutral and angry expressions.

Another explanation for the motor deficits of child with ASD is the failure in visual and motor circuitry. Stereotyped and repetitive behaviors are reported in $64 \%$ of the ASD cases and the child realizes the inadequate movements mainly in the attempt of sensory regulation as a mechanism of an organization [31]. In ASD, proprioceptive prejudice associated with reception failure and visual stimuli processing harms motor learning and contributes to the motor behavior and social inappropriateness [32], facilitating the presence of the stereotyped and repetitive behavior. The sensory processing disorder (hypo- or hyperresponsive) may be related to the origin of the functional limitations of the child [33]. Also, there might be some prejudice in motor planning because of the inappropriate visual processing in the ASD. Knowing that the motor and sensory systems cooperate with each other [34], the joint failure of these systems can cause motor dysfunction. A second possible explanation for this study having verified more activation of fast frequencies in the center region is neural plasticity. In children with ASD, failure in sensory/visual processing and in planning together can promote differentiated motor behavior that might occur due to the activation of groups of neurons of primary function without regulation. ASD children might have a failure in the visual processing because of a modification in the communication between dorsal and ventral pathways, which are mediated by connections with the frontal cortex [27]. The failure of the visual processing can promote more activation in the primary visual area.

In this study, a high activation in the occipital areas was found. Bigger activation in $\mathrm{O} 1$ and $\mathrm{O} 2$ that occurred when happy and angry face group were presented might be due to stronger activation of the primary visual cortex relative to the control group. It is known that in ASD there are failures in visual perception and that it seems to cause more fixation to parts of the stimulus relative to the whole, and also there are erratic visualization patterns [35]. This more intense focal fixation and probable prejudice of face processing can justify the observed pattern. Maybe in the neutral face, the pattern did not occur because there were no "distractors" such as the 

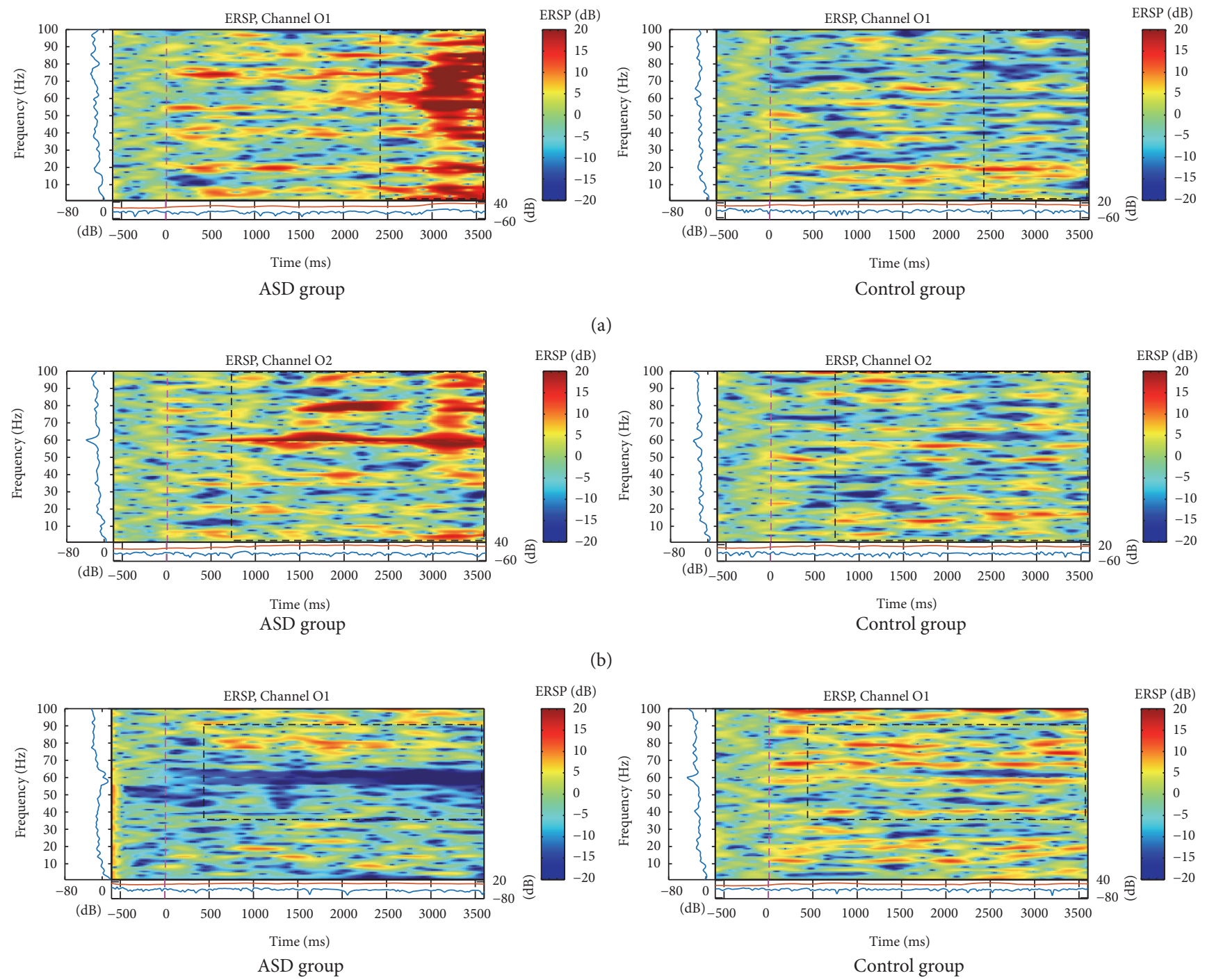

(b)

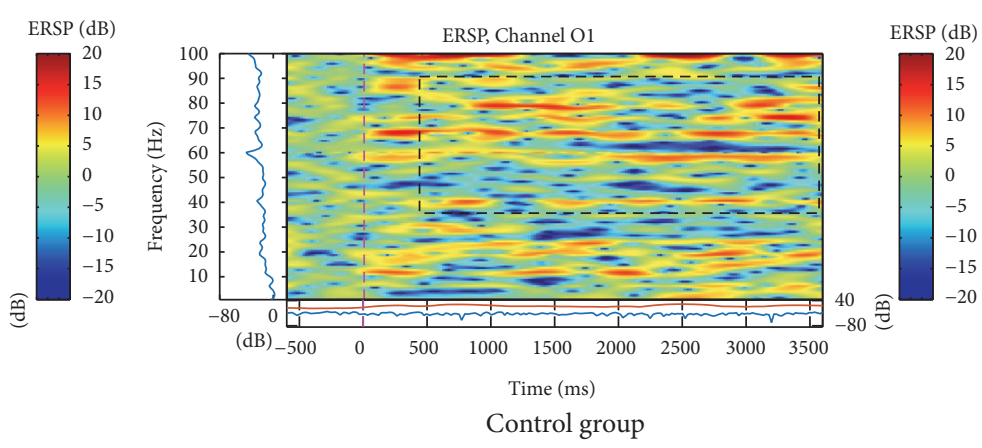

(c)

FIGURE 7: ERSP of (a) and (c) O1 and (b) O2 electrodes for the total mean of the samples of the happy (a) and (b) and neutral (c) faces signals in the interval of 3 seconds (plus a basal activity of $500 \mathrm{~ms}$ before the event) for the ASD and control groups that showed significant spectral perturbations. In the ERSP image, the upper panel presents the ERSP (Event-Related Spectral Perturbation) data in $\mathrm{dB}$, with mean baseline spectral power (in $\mathrm{dB}$ ) subtracted at each time in the epoch. The upper left marginal panel presents the mean spectral power during the baseline period (blue). The marginal panel under the ERSP image shows the maximum (red) and minimum (blue) ERSP values relative to baseline power at each frequency.

muscular contraction that occurs in other expressions which attract more focal attention.

\section{Conclusion}

The analysis of the power spectrum in children with ASD during visual stimulus of happy, neutral, and angry faces demonstrated an increase of power in higher frequencies (above $30 \mathrm{~Hz}$ ) in the ASD group in frontal, occipital, and center-parietal areas when compared to control group. More studies are needed to better understand these differences.

\section{Conflicts of Interest}

The authors declare that they have no conflicts of interest.

\section{Acknowledgments}

The authors would like to thank the parents and kids from both groups: ASD and control, who were volunteers for this study, the technician Luciana de Morais Batista Silva who helped in data acquisition, the neuropsychologist Samantha Santos de Albuquerque Maranhão who evaluated the children and participated in the discussions, the CEPS, Jackson 

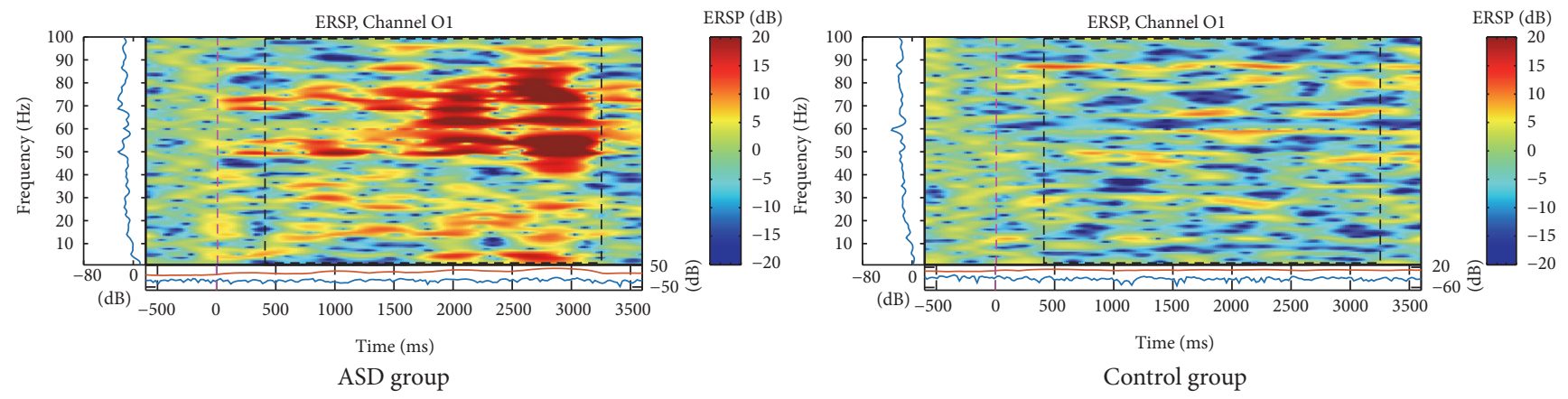

(a)
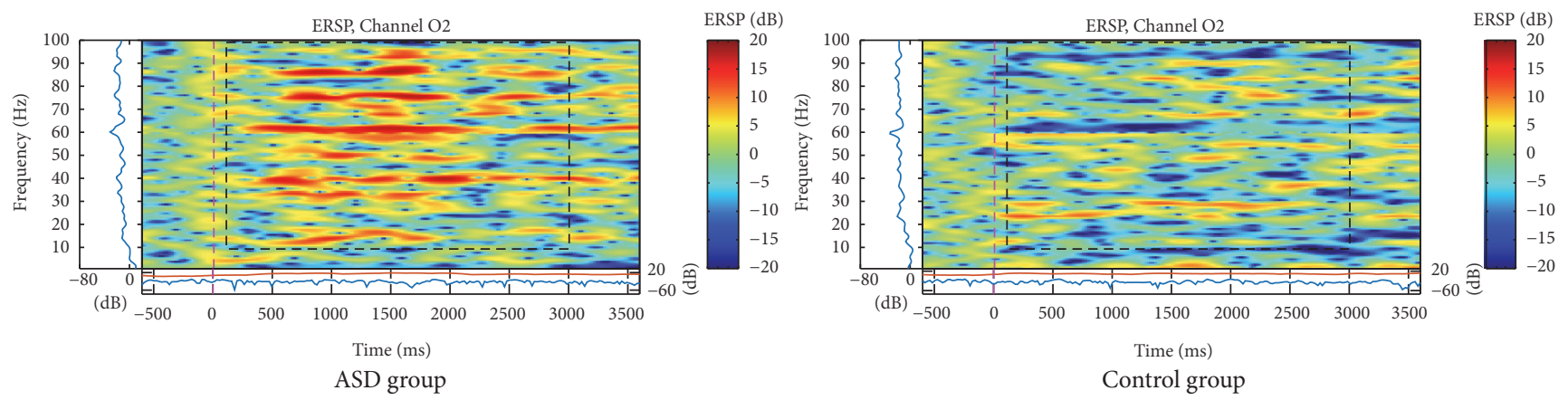

(b)

FIGURE 8: ERSP of (a) O1 and (b) O2 electrodes for the total mean of the samples of the angry faces signals in the interval of 3 seconds (plus a basal activity of $500 \mathrm{~ms}$ before the event) for the ASD and control groups that showed significant variations or spectral perturbations. In the ERSP image, the upper panel presents the ERSP (Event-Related Spectral Perturbation) data in dB, with mean baseline spectral power (in $\mathrm{dB}$ ) subtracted at each time in the epoch. The upper left marginal panel presents the mean spectral power during the baseline period (blue). The marginal panel under the ERSP image shows the maximum (red) and minimum (blue) ERSP values relative to baseline power at each frequency.

Cionek from Brain Support for the eye-tracking system support, Professors Sanjay Joshi and Kenneth Lyons from UC Davis RASCAL Lab for the final review, and Brazilian Ministry of Education (MEC).

\section{References}

[1] American Psychiatric Association, Diagnostic and Statistical Manual of Mental Disorders: DSM IV, American Psychiatric Association, Washington, Wash, USA, 4th edition, 1994.

[2] American Psychiatric Association, Diagnostic and Statistical Manual of Mental Disorders, text rev- DSM IV-TR, American Psychiatric Press, Washington, Wash, USA, 4th edition, 2000.

[3] L. Kanner, "Autistic disturbances of affective contact," Nervous Child, vol. 2, pp. 217-250, 1943.

[4] L. Y. Tsai, M. C. Tsai, and G. J. August, "Brief report: Implication of EEG diagnoses in the subclassification of infantile autism," Journal of Autism and Developmental Disorders, vol. 15, no. 3, pp. 339-344, 1985.

[5] J. S. Schwartzman and C. A. Araújo, Transtorno do Espectro do Autismo, MEMNON Edições Científicas, São Paulo, Brazil, 2011.

[6] M. T. Roelfsema, R. A. Hoekstra, C. Allison et al., "Are autism spectrum conditions more prevalent in an informationtechnology region? A school-based study of three regions in the
Netherlands," Journal of Autism and Developmental Disorders, vol. 42, no. 5, pp. 734-739, 2012.

[7] J. Wang, J. Barstein, L. E. Ethridge, M. W. Mosconi, Y. Takarae, and J. A. Sweeney, "Resting state EEG abnormalities in autism spectrum disorders," Journal of Neurodevelopmental Disorders, vol. 5, no. 1, article 24, 2013.

[8] E. Fombonne, "Epidemiology of pervasive developmental disorders," Pediatric Research, vol. 65, no. 6, pp. 591-598, 2009.

[9] H. I. Kaplan and B. J. Sadock, Compêndio de Psiquiatria, Artes Médicas, Porto Alegre, Brazil, 1990.

[10] M. C. Christina, "Early Intervention in autismo," Infants and Young Children, vol. 18, no. 2, pp. 74-85, 2005.

[11] A. M. Pereira, Autismo infantil: tradução e validação da CARS (childhood autismo ratinh scale) para uso no Brasil, Dissertação de Mestrado, Porto Alegre, Brazil, 2007.

[12] S. Bölte, K. D. Bartl-Pokorny, U. Jonsson et al., "How can clinicians detect and treat autism early? Methodological trends of technology use in research," Acta Paediatrica, International Journal of Paediatrics, vol. 105, no. 2, pp. 137-144, 2016.

[13] S. J. Spence and M. T. Schneider, "The role of epilepsy and epileptiform eegs in autism spectrum disorders," Pediatric Research, vol. 65, no. 6, pp. 599-606, 2009.

[14] N. N. Boutros, R. Lajiness-O'Neill, A. Zillgitt, A. E. Richard, and S. M. Bowyer, "EEG changes associated with autistic spectrum disorders," Neuropsychiatric Electrophysiology, vol. 1, no. 1, 2015.

[15] H. Sheikhania, B. M. R. Behnam, C. Mohammadi, and D. M. Noroozian, "Analysis of EEG background activity in Autism 
disease patients with bispectrum and STFT measure," in Proceedings of the 11th WSEAS International Conference on COMMUNICATIONS, Agios Nikolaos, Crete Island, Greece, July 2007.

[16] M. Hashemian and H. Pourghassem, "Diagnosing autism spectrum disorders based on EEG analysis: A survey," Neurophysiology, vol. 46, no. 2, pp. 183-195, 2014.

[17] M. M. Ravicz, K. L. Perdue, A. Westerlund, R. E. Vanderwert, and C. A. Nelson, "Infants' neural responses to facial emotion in the prefrontal cortex are correlated with temperament: a functional near-infrared spectroscopy study," Frontiers in Psychology, vol. 6, article 922, 2015.

[18] A. L. Tierney, L. Gabard-Durnam, V. Vogel-Farley, H. TagerFlusberg, and C. A. Nelson, "Developmental trajectories of resting eeg power: an endophenotype of autism spectrum disorder," PLoS ONE, vol. 7, no. 6, Article ID e39127, 2012.

[19] J. N. Saby and P. J. Marshall, "The utility of EEG band power analysis in the study of infancy and early childhood," Developmental Neuropsychology, vol. 37, no. 3, pp. 253-273, 2012.

[20] C. Akasari and S. Pattersom, "Interventions Addressing Social Impairment in Autism," Current Psychiatry Reports, vol. 14, no. 6, pp. 713-725, 2012.

[21] N. B. Pitskel, D. Z. Bolling, M. D. Kaiser, K. A. Pelphrey, and M. J. Crowley, "Sistemas neurais de reavaliação cognitiva em crianças e adolescentes com transtorno do espectro do autismo," Developmental Cognitive Neuroscience, vol. 10, pp. 117-128, 2014.

[22] A. N. Meltzoff and M. K. Moore, "Explaining facial imitation: a theoretical model," Early Development and Parenting, p. 179, 1997.

[23] S. Hanawa, M. Sugiura, T. Nozawa et al., "The neural basis of the imitation drive," Social Cognitive and Affective Neuroscience, vol. 11, no. 1, Article ID nsv089, pp. 66-77, 2015.

[24] I. Puzzo, N. R. Cooper, P. Vetter, R. Russo, and P. B. Fitzgerald, "Reduced cortico-motor facilitation in a normal sample with high traits of autism," Neuroscience Letters, vol. 467, no. 2, pp. 173-177, 2009.

[25] I. Puzzo, N. R. cooper, P. Vetter, and R. Russo, "EEG diferenças de ativação no córtex pré- motor e área motora suplementar entre indivíduos normais com traços altos e baixos de autismo," Brain Research, vol. 1342, pp. 104-110, 2010.

[26] D. L. Floris, A. D. Barber, M. B. Nebel et al., "Atypical lateralization of motor circuit functional connectivity in children with autism is associated with motor deficits," Molecular Autism, vol. 7, no. 1, article no. 35, 2016.

[27] T. P. Deramus, B. S. Black, M. R. Pennick, and R. K. Kana, "Enhanced parietal cortex activation during location detection in children with autism," Journal of Neurodevelopmental Disorders, vol. 6, no. 1, article no. 37, 2014.

[28] J. B. Wagner, S. B. Hirsch, V. K. Vogel-Farley, E. Redcay, and C. A. Nelson, "Eye-tracking, autonomic, and electrophysiological correlates of emotional face processing in adolescents with autism spectrum disorder," Journal of Autism and Developmental Disorders, vol. 43, no. 1, pp. 188-199, 2013.

[29] K. Pierce, S. Marinero, R. Hazin, B. McKenna, C. C. Barnes, and A. Malige, "Eye tracking reveals abnormal visual preference for geometric images as an early biomarker of an autism spectrum disorder subtype associated with increased symptom severity," Biological Psychiatry, vol. 79, no. 8, pp. 657-666, 2016.

[30] L. A. Kimberly, C. D. Gilman, J. Meinzen-Derr et al., "Multifrequency localization of aberrant brain activity in autism spectrum disorder," Brain and Development, vol. 38, no. 1, pp. 82-90, 2015.
[31] A. M. Amiri, N. Peltier, C. Goldberg et al., "WearSense: detecting autism stereotypic behaviors through smartwatches," Saúde, vol. 5, no. 1, p. 11, 2017.

[32] M. B. Nebel, S. E. Joel, J. Muschelli et al., "Disruption of functional organization within the primary motor cortex in children with autism," Human Brain Mapping, vol. 35, no. 2, pp. 567-580, 2014.

[33] E. Gal, M. J. Dyck, and A. Passmore, "Relationships between Stereotyped Movements and sensory processing disorders in children with and without developmental or sensory disorders," American Journal of Occupational Therapy, vol. 64, no. 3, pp. 453-461, 2010.

[34] A. Tomassini, D. Spinelli, M. Jacono, G. Sandini, and M. C. Morrone, "Rhythmic oscillations of visual contrast sensitivity synchronized with action," Journal of Neuroscience, vol. 35, no. 18, pp. 7019-7029, 2015.

[35] G. Golarai, K. Grill-Spector, and A. L. Reiss, "Autism and the development of face processing," Clinical Neuroscience Research, vol. 6, no. 3-4, pp. 145-160, 2006. 

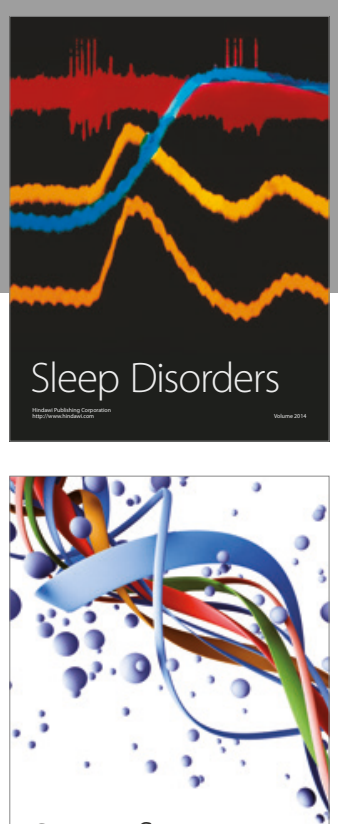

Scientifica
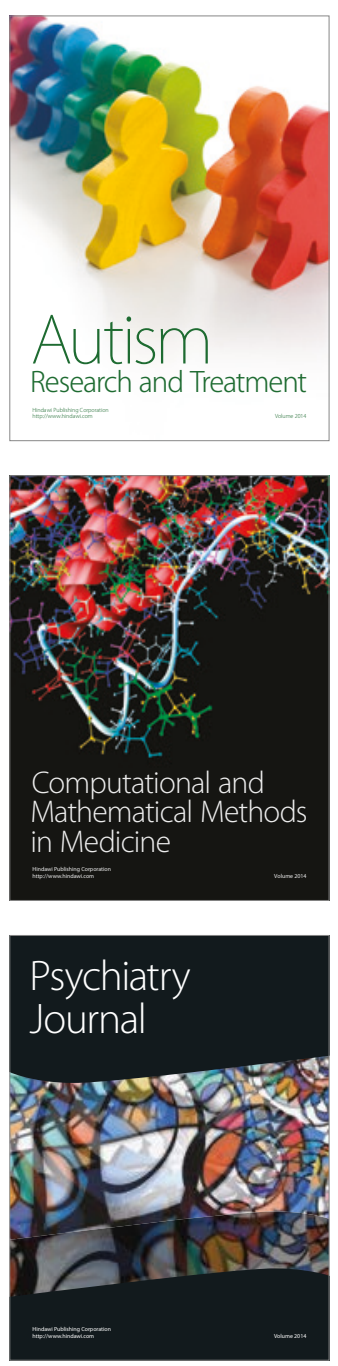
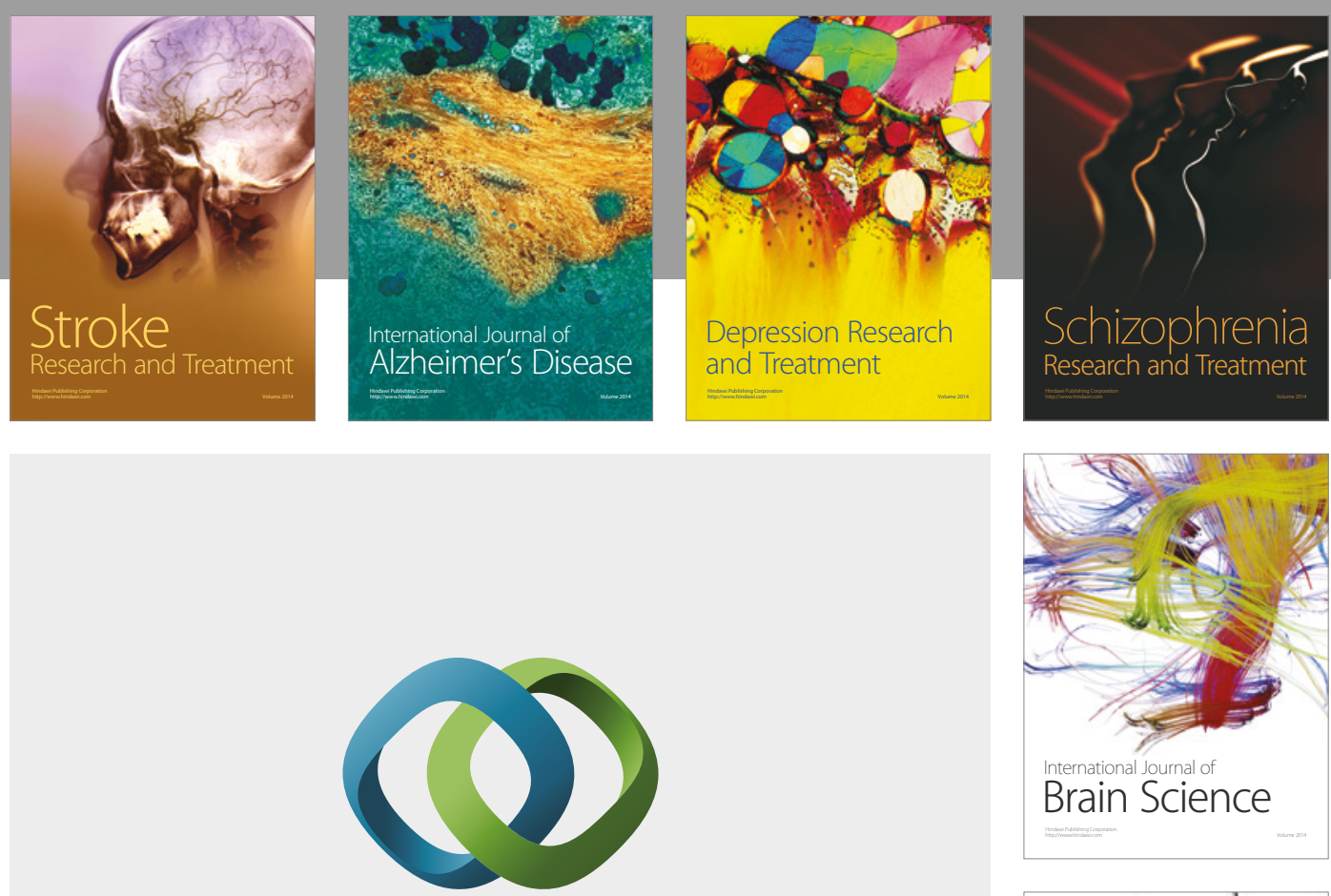

\section{Hindawi}

Submit your manuscripts at

https://www.hindawi.com
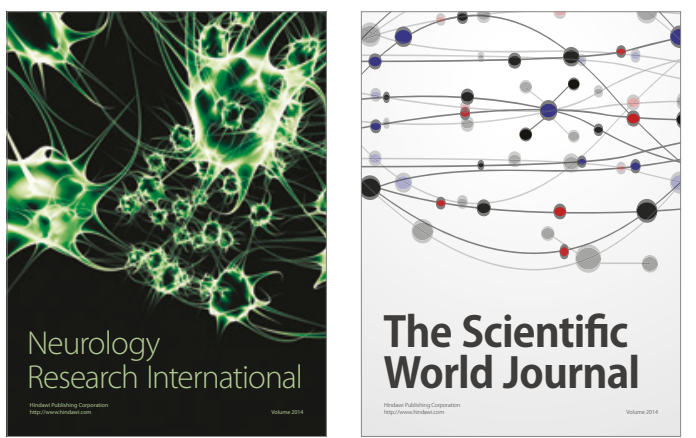

The Scientific World Journal

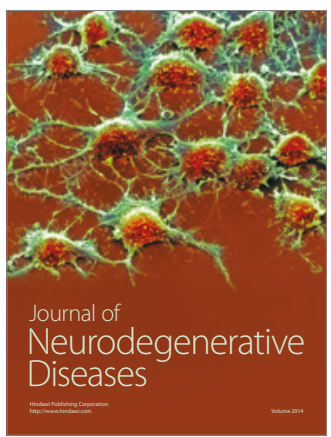

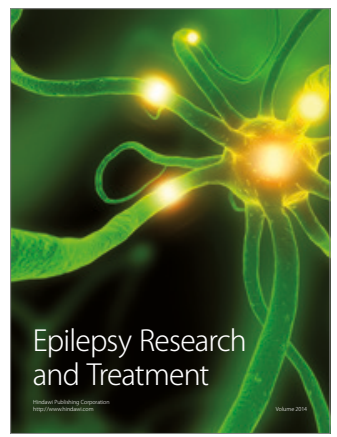

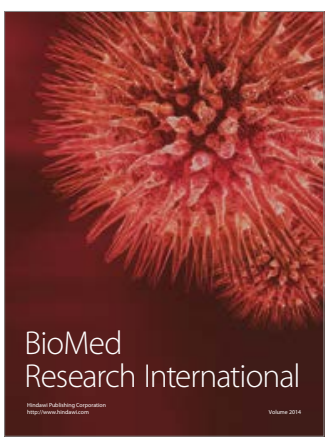

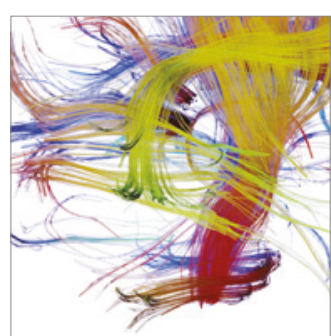

Brain Science

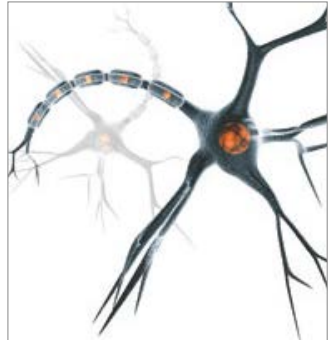

Neural Plasticity
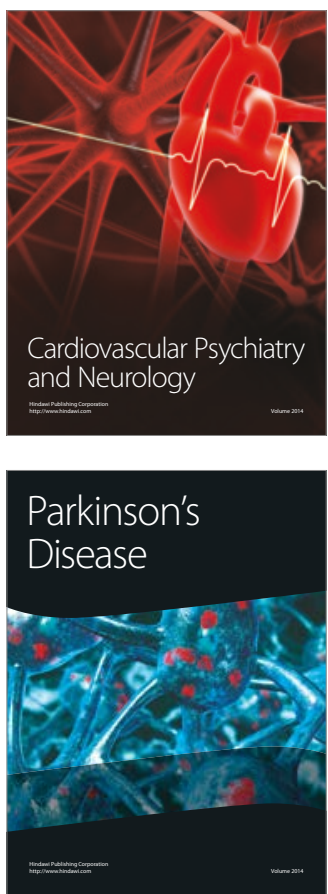